\title{
Composite cylindrical shells under combined loading
}

\author{
Panagiotis Charitidis ${ }^{1, *}$, Dimitrios Zacharopoulos ${ }^{2}$ \\ ${ }^{1}$ Democritus University of Thrace, Environmental Engineering School, 67 100, Xanthi, Greece \\ ${ }^{2}$ Democritus University of Thrace, Engineering School, 67 100, Xanthi, Greece
}

\begin{abstract}
The structural design of a cylindrical shell made of a unidirectional glass/epoxy fiber composite and subjected to torsional moment and internal pressure is studied. An anisotropic model for analysis of composite material is applied. The minimum wall thickness of the shell, with the use of the Tsai-Hill failure criterion, is derived. In this connection, the strain energy density criterion (SED) is applied, by which the critical crack length is determined. In this paper, we illustrate the fracture methodology towards the design of a cylindrical shell structure for determining safe operation. Finite element analysis and analytical method were also established.
\end{abstract}

\section{Introduction}

Continuous-fiber composite materials have gained popularity in modern structures during the past few years due to their flexibility in obtaining the desired mechanical and physical properties in combination with light-weight components. Unfortunately, the fracture of orthotropic materials differs significantly from the fracture of isotropic materials. It means, that the mathematical principles involved are much more complex. In a "previous" paper [1] we analyzed the problem of a cracked anisotropic elastic plate subjected to a load that is oriented at an arbitrary angle with the crack direction which does not coincide with the principal axes of material symmetry of the plate. The crack growth angle and the critical load at crack instability were determined by strain energy density theory. In a subsequent paper [2], the mechanical behavior of a cylindrical shell made of fiber composite material was studied. In this study, a thick-walled cylinder is examined. A thick-walled cylinder is a pressure vessel whose wall thickness is greater than one-tenth of its inner radius [3], while the theory of thick tubes (Lame's theory) shows that the longitudinal cracks located on the internal face of the cylinder are most dangerous [4]. The length of a crack in a pressure vessel may increase due to the application of repeated loads or due to a combination of loads and environmental attack. As its length increases, so does the stress concentration induced by it [5]. Due to the presence of the crack, the residual strength of the pressure vessel decreases progressively with increasing crack size until it becomes so low that the pressure vessel cannot withstand high pressures. Even if the cylinder is not subjected to very high pressures, the crack will continue to grow until the residual strength becomes so low that fracture occurs under normal service pressure [5]. Cracks may result in leakage or rupture failures. Furthermore, a finite element analysis has also been used. Simulating the propagation of cracks by using finite element method is quite challenging because the topology of the domain changes continuously. For this reason, the extended finite element method (X-FEM) is being

\footnotetext{
${ }^{1}$ Corresponding author: nanocomp@mail.com
} 
used to model cracks because the mesh can be created independent from the crack geometry. Actually, X-FEM lies in applying the appropriate enrichment function locally in the domain of interest using the partition of unity.

In the present work a cylindrical shell [6], made of glass/epoxy continuous-fiber composite with designation Scotchply 1002 under torsion and internal pressure loadings, is assumed. The fiber composite material is modeled as a homogeneous orthotropic continuous medium [7]. Determination of the minimum required shell wall thickness was achieved by Tsai-Hill failure criterion. After that, the methodology of elastic fracture mechanics was applied. The stress components in the vicinity of the crack tip are obtained in terms of two stress intensity factors [8]. The results of stress analysis are combined with the strain energy density criterion $[9,10]$ to obtain the critical crack length for shell wall thickness and fiber direction.

\section{Theoretical considerations}

\subsection{Stress field in crack in anisotropic plate under general in-plane loadings}

It is well known, that the existence of cracks in structural materials creates a need for the understanding of stress distributions at the tip of these cracks. In this study, consider a homogeneous rectilinearly orthotropic material plate, whose principal axes of material symmetry coincide with the $\mathrm{x}$ and $\mathrm{y}$ directions (Fig. 1a, b). A through crack of length $2 \mathrm{a}$ in the material element is assumed, making an angle $\varphi$ with the Ox axis. The plate is subjected to the normal $\sigma_{1}, \sigma_{2}$ and the shear $\tau$ stresses with respect to the system $\mathrm{O}_{\mathrm{x}^{\prime \prime} \mathrm{y}^{\prime \prime}} \mathrm{O}_{\mathrm{x}} \mathrm{y}$. The stress field $[1,2,7,8]$ in the vicinity of the crack tip with respect to system $\mathrm{O}_{\mathrm{x}^{\prime \prime} \mathrm{y}^{\prime \prime}} \mathrm{O}_{\mathrm{x}} \mathrm{y}$, as well as, the material properties $\mu_{1}$ and $\mu_{2}$ is given by,

$$
\sigma_{\chi}=\frac{K_{1}}{\sqrt{2 \pi r}} A+\frac{K_{2}}{\sqrt{2 \pi r}} B, \sigma_{y}=\frac{K_{1}}{\sqrt{2 \pi r}} C+\frac{K_{2}}{\sqrt{2 \pi r}} D, \tau_{\chi y}=\frac{K_{1}}{\sqrt{2 \pi r}} E+\frac{K_{2}}{\sqrt{2 \pi r}} F
$$

where

$$
\begin{aligned}
& A=\operatorname{Re}\left[\frac{s_{1} s_{2}}{s_{1-} S_{2}}\left(\frac{s_{2}}{z_{2}}-\frac{s_{1}}{z_{1}}\right)\right], B=\operatorname{Re}\left[\frac{1}{s_{1-} s_{2}}\left(\frac{s_{2}^{2}}{z_{2}}-\frac{s_{1}^{2}}{z_{1}}\right)\right] \\
& C=\operatorname{Re}\left[\frac{1}{s_{1-} s_{2}}\left(\frac{s_{1}}{z_{2}}-\frac{s_{2}}{z_{1}}\right)\right], D=\operatorname{Re}\left[\frac{1}{s_{1-} s_{2}}\left(\frac{1}{z_{2}}-\frac{1}{z_{1}}\right)\right] \\
& E=\operatorname{Re}\left[\frac{s_{1} s_{2}}{s_{1-} s_{2}}\left(\frac{1}{z_{2}}-\frac{1}{z_{1}}\right)\right], F=\operatorname{Re}\left[\frac{1}{s_{1-} s_{2}}\left(\frac{s_{1}}{z_{2}}-\frac{s_{2}}{z_{1}}\right)\right]
\end{aligned}
$$

and complex variables $\mathrm{z}_{1}$ and $\mathrm{z}_{2}$ are defined by

$$
z_{1}=\left(\cos \theta+s_{1} \sin \Theta\right), z_{2}=\left(\cos \theta+s_{2} \sin \Theta\right)
$$

In these equations the symmetric and skew-symmetric stress intensity factors $K_{I}$ and $K_{2}$ are expressed by 


$$
\begin{gathered}
K_{1}=\left(\gamma \cos ^{2} \beta+\sin ^{2} \beta-\eta \sin 2 \beta\right) \sigma_{2} \sqrt{\pi \alpha} \\
K_{2}=0,5[(1-\gamma) \sin 2 \beta-2 \eta \cos 2 \beta) \sigma_{2} \sqrt{\pi \alpha}
\end{gathered}
$$

where $\beta$ is the angle that the crack axis subtends with the direction of stress $\sigma_{2}$. The complex parameters $\mu_{1}$ and $\mu_{2}$, are the roots of characteristic equation

$$
a_{11} \mu^{4}-2 a_{16} \mu^{3}+\left(2 a_{12}+a_{66}\right) \mu+a_{22}=0
$$

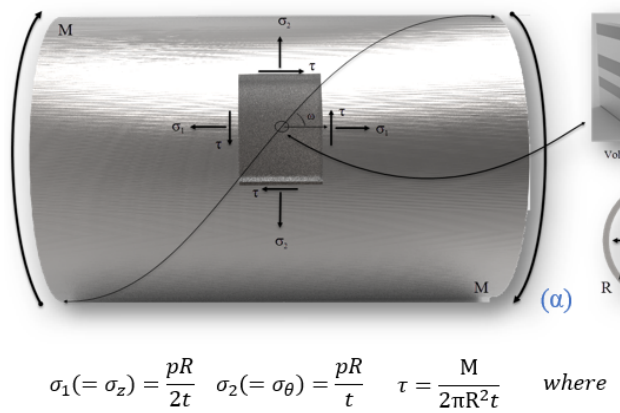

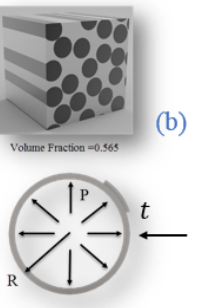

$t$ : thickness $\mathrm{R}$ : radius $P$ : pressure $M$ : torque

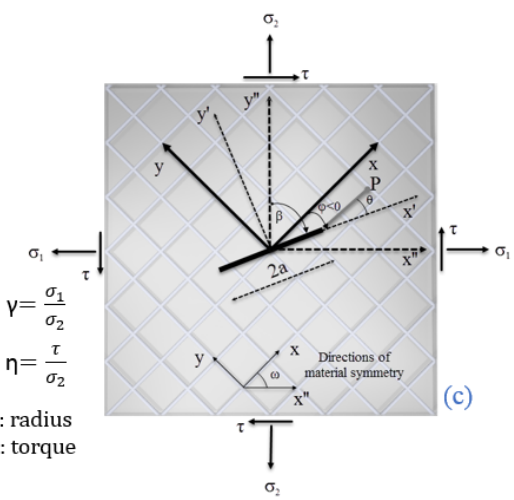

Fig. 1. Composite cylinder under combined loading.

where the constants of the material $\alpha_{\mathrm{kl}}^{\prime}$ are referred to the system $\mathrm{O}_{\mathrm{x}^{\prime} \mathrm{y}^{\prime}}$. From equation (4) it was obtained for $\mu_{1}$ and $\mu_{2}$ that

$$
\mu_{1}=3.2035 \mathrm{i}, \mu_{2}=0.6486 \mathrm{i}
$$

\subsection{Failure Criteria}

To analyze the strength of any laminated composite, strength theories are required. In literature, there are numerous failure criteria for composite materials as a result of the complex nature of observed failure phenomena. Now, failure criteria for composite materials are often classified into two categories, the non-interactive and interactive failure criteria. The maximum stress and maximum strain criteria are non-interactive failure criteria, while Tsai Hill, Tsai Wu, Hashin's, Puck's, and Christensen's are interactive failure criteria. Failure envelopes for the above criteria were created for $0^{\circ}$ and $46.5^{\circ}$ fiber orientation (figure $2 \mathrm{a}-\mathrm{f}$ ), respectively. Laminate failure is the eventual result of progressive failure processes taking place in the constituent laminae under loading. It should be noted, that the direction $\mathrm{x}^{\prime \prime}$ is parallel to the axis of the shell and $\mathrm{x}$ coincides with the fiber direction. The angles $\omega, \varphi$ and $\beta$ are related by the equation $\omega+\varphi+\beta=90^{\circ}$.

In this study, the strain energy density fracture criterion was chosen. The basic quantity in strain energy density theory is the strain energy function, $\mathrm{dW} / \mathrm{dV}$ which, even for a rectilinearly anisotropic material, can be put in the form 


$$
\frac{d W}{d V}=\int_{0}^{\varepsilon_{i j}} \sigma_{i j} d \varepsilon_{i j}=\frac{S}{r}
$$

where $r$ is the distance of element, from the crack tip and $S$ the strain energy density factor. The fundamental hypotheses on unstable crack growth for brittle materials in the energy density theory $(1,8)$, are as follows:

(1) Crack initiation takes place in a direction coinciding with the maximum of the strain energy density factor among stationeries minima, i.e,

$$
\theta_{c}=\max \left\{\frac{\theta}{\frac{\partial S}{\partial \theta}}\right\}=0, \quad \frac{\partial^{2} S}{\partial \theta^{2}}>0
$$

(2) Unstable crack growth, occurs when the strain energy density factor reaches a critical value, i.e.,

$$
S_{\min }^{\max }\left(\theta_{c}\right)=S_{c}
$$

The strain energy density factor $\mathrm{S}$ is computed from equations (1), (5) and the expression

$$
\frac{d W}{d V}=\frac{1}{2}\left(\sigma_{x} \varepsilon_{x}+\sigma_{y} \varepsilon_{y}+\tau_{x y} \gamma_{x y}\right)
$$

It is obtained for the case of generalized plane stress that

$$
S=A_{11} k_{1}^{2}+2 A_{12} k_{1} k_{2}+A_{22} k_{2}^{2}
$$

The coefficients $A_{11}, A_{12}$ and $A_{22}$ are implicit functions of the elastic constants $\alpha_{\mathrm{kl}}^{\prime}$ and depend on the angle $\theta$ through $\mathrm{z}_{1}$ and $\mathrm{z}_{2}$.

\section{Material Properties}

The objective is to design a cylindrical shell made of a glass/epoxy continuous fiber reinforced composite, where the direction of reinforcement is at an angle $\omega$ with the longitudinal axis of the shell (Figure 1a). The shell has a nominal radius $\mathrm{R}=500 \mathrm{~mm}$ and is subjected to an internal pressure $\mathrm{p}$ and a torsional moment $\mathrm{M}$. Let $\mathrm{t}$ denote the wall thickness of the shell. Then, the normal $\sigma_{1}\left(=\sigma_{\theta}\right), \sigma_{2}\left(=\sigma_{z}\right)$ and shear $\tau$ stresses developed in the shell (figure 1a-c).

A through the shell wall crack of length 2 a, whose axis makes an angle $\left(90^{\circ}-\beta\right)$ with the longitudinal axis of the shell, is assumed. The glass/epoxy unidirectional fiber reinforced composite of which the shell is made, has the commercial designation Scotchply 1002. The engineering properties of this material are [11]

$$
E_{1}=40.0 \mathrm{GPa}, \mathrm{E}_{2}=8.3 \mathrm{GPa}, \mathrm{G}_{12}=4.1 \mathrm{GPa}, v_{12}=0.26, v_{23}=0.34
$$


The strengths of Scotchply 1002, are on principal directions of the material.

$$
\mathrm{X}=770.0 \mathrm{MPa}, \mathrm{Y}=22.0 \mathrm{MPa}, \mathrm{T}=30.0 \mathrm{MPa}
$$

The fracture toughness of Scotchply 1002 for different angle $\varphi(\mathrm{rad})$ of the crack to the fiber orientations, has determined [6] by the following empirical formula

$$
\mathrm{K}_{\mathrm{Ic}}(\varphi)=1.086 \varphi+0.279 \varphi+1.469,\left(\mathrm{MPam}^{1 / 2}\right)
$$
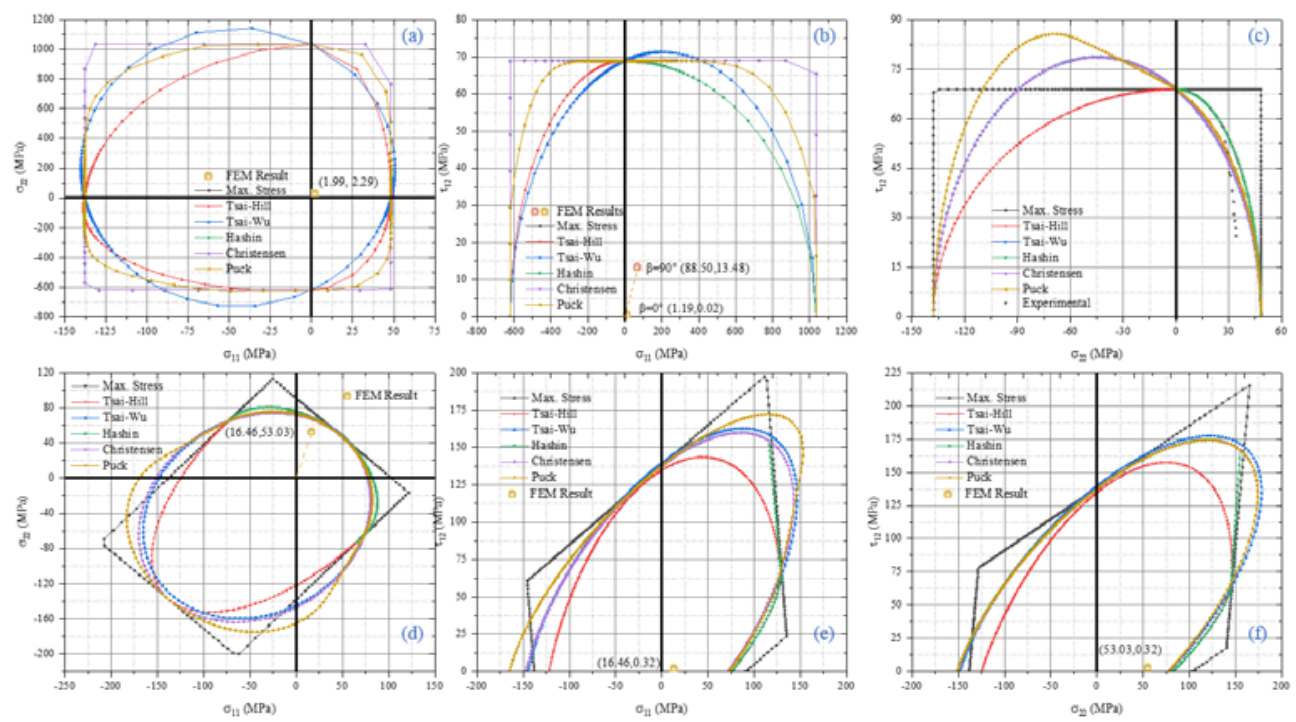

Fig. 2. Failure envelopes for $0^{\circ}$ (a-c) and $46.50^{\circ}$ (d-f) angle for Scotchply-1002.

\section{Structural design of cylindrical shell}

The minimum necessary thickness of the shell wall, $t_{\mathrm{cr}}$, when the shell is subjected to internal pressure $\mathrm{p}$ and torsional moment $\mathrm{M}$ according to the strain energy density criterion, is computed from the expression

$$
\bar{S}\left(t_{c r}, a, \omega, \beta\right)=S_{c}(\varphi)
$$

where $\overline{\mathrm{S}}$ is a function, of the length of the crack, $2 \mathrm{a}$, the angle of propagation $\theta_{\mathrm{c}}$, the angle $\omega$ of direction of the fiber with the axis of the shell and $\beta$, the angle of the axis of the crack with axis $\mathrm{y}^{\prime \prime}$. According to Tsai-Hill failure criterion the minimum necessary shell wall thickness $t_{c r}$ is given by the relation

$$
t_{c r}=p^{2} R^{2} Z\left(\omega, \sigma_{1}, \sigma_{2}, \tau, X, Y, T\right)
$$

where $\mathrm{Z}$ is a function of the angle $\omega$ and stresses $\sigma_{1}, \sigma_{2}, \tau$ and material strengths $\mathrm{X}, \mathrm{Y}$ and T. Figure $3 a$ present the variation of critical thickness $t_{\text {cr }}$ versus the angle $\omega$ with dashed and point line according to Tsai-Hill criterion. The shell was subjected only to internal pressure $(\gamma=0.5, \eta=0.0)$. Observe that the minimum critical thickness for structural design of the shell is $t_{\mathrm{cr}}=6.8 \mathrm{~cm}$, when the direction of the fiber is perpendicular to the longitudinal axis of shell. The critical length $2 \mathrm{a}$ of the crack against $t_{c r}$ is given in Figure $3 a$ with 
continuous line, according to the criterion of the SED theory. Then the thickness $t_{\mathrm{cr}}=6.8 \mathrm{~cm}$ and $\beta=0^{\circ}$ is chosen, the critical length of the crack is $2 a_{c}=0.29 \mathrm{~cm}$.

Analogous results, when internal pressure $\mathrm{p}=6 \mathrm{MPa}$ and torsional moment $\mathrm{M}=2.12$ $\mathrm{MNm}$ are applied, are presented in figure $3 \mathrm{~b}$. Observe that the favorable case is achieved when the fiber angle is $\omega=46.5^{\circ}$. The critical thickness for this direction of the fiber is $t_{c r}=4.8$ $\mathrm{cm}$, according to Tsai-Hill criterion. Then the critical length of the crack, according to the strain energy density criterion is $2 \mathrm{a}_{\mathrm{c}}=0.12 \mathrm{~cm}$, when $\beta=90^{\circ}$. Figure $3 \mathrm{c}$ presents the variation of the angle $v$ versus angle $\beta$ for values of the angle $\omega=0^{\circ}, 45^{\circ}$ and $90^{\circ}$, when the shell is subjected to internal pressure only. The direction of crack growth makes an angle $v$ with the direction of the reinforcing fiber. This figure shows that, regardless of its original direction, crack propagation moves parallel to the fiber direction; this result is corroborated in practice.

\section{Finite Element Analysis}

Three-dimensional finite element analysis has been conducted in order to analyze the behavior of the orthotropic cylinder under internal pressure and moment. The calculation of the SIF is carried out in two steps. Firstly, a global finite element (FE) model of the whole cylinder without crack is generated. The result of this step of the analysis is the stress-strain state at the positions where a crack initiation from a defect would be likely. Then, the JIntegral is calculated in a sub-model (figure $4 \mathrm{a}$ ), in order to obtain more accurate results. The contour integral calculation is the most important aspect in the stationary crack analysis since it gives the measure to assess the critical crack size (figure $4 \mathrm{~b}$ ). The stress results were plotted into failure envelopes, to be able to compare these values with the results of the failure criteria.

Before apply X-FEM technique, the crack and the crack domain should be defined and chosen, respectively. The crack domain defines where the enrichment features can be added to the finite element approximation, i.e. the region where a crack can be described with XFEM. It is specified manually and for stationary crack analyses it must contain any existing crack. The enrichment radius is a radius from the crack tip and defines in which element nodes the crack tip functions are added. Furthermore, it can be manually chosen or Abaqus calculates the radius as three times the typical element characteristic length. This characteristic length is a typical length of a line across an element. The radius is chosen to be calculated automatically in all models presented in the stationary crack evaluation. The last step is to specify the number of contours to be included in the contour domain to calculate the stress intensity factors. Moreover, PHILSM and PSILSM must be selected in the Field Output Requests to be able to visualize the crack opening in the post-process. These parameters are non-zero, only for the enriched elements (figure $4 b$ ).

\section{Results}

Based on the previous development results concerning the failure behavior of the cylindrical shell containing an initial crack and made of a unidirectional fiber composite were obtained. In the first case, the shell was subjected only to internal pressure $(\gamma=0.5, \eta=0)$ and the direction of fiber reinforcement was parallel to the longitudinal axis of the shell $(\omega=0)$. As it can be seen, from the above results, critical stress $\sigma_{2 \text { cr }}$ for crack growth decreases with the angle $\beta$ and takes its minimum value when the crack is oriented along the circumferential direction of the shell. Furthermore, it is well known, that $\sigma_{2 \text { cr }}$ increases as the fiber volume ratio to composite $\mathrm{V}_{\mathrm{f}}$ also increases. It should be noted that the volume fraction that appears in figure 1 , is only a representative figure. In the second case, it is observed that takes its maximum value at an angle different than $\beta=90^{\circ}$ in the interval $0<\beta<90^{\circ}$. At this angle the 
critical stress for crack growth takes its minimum value.
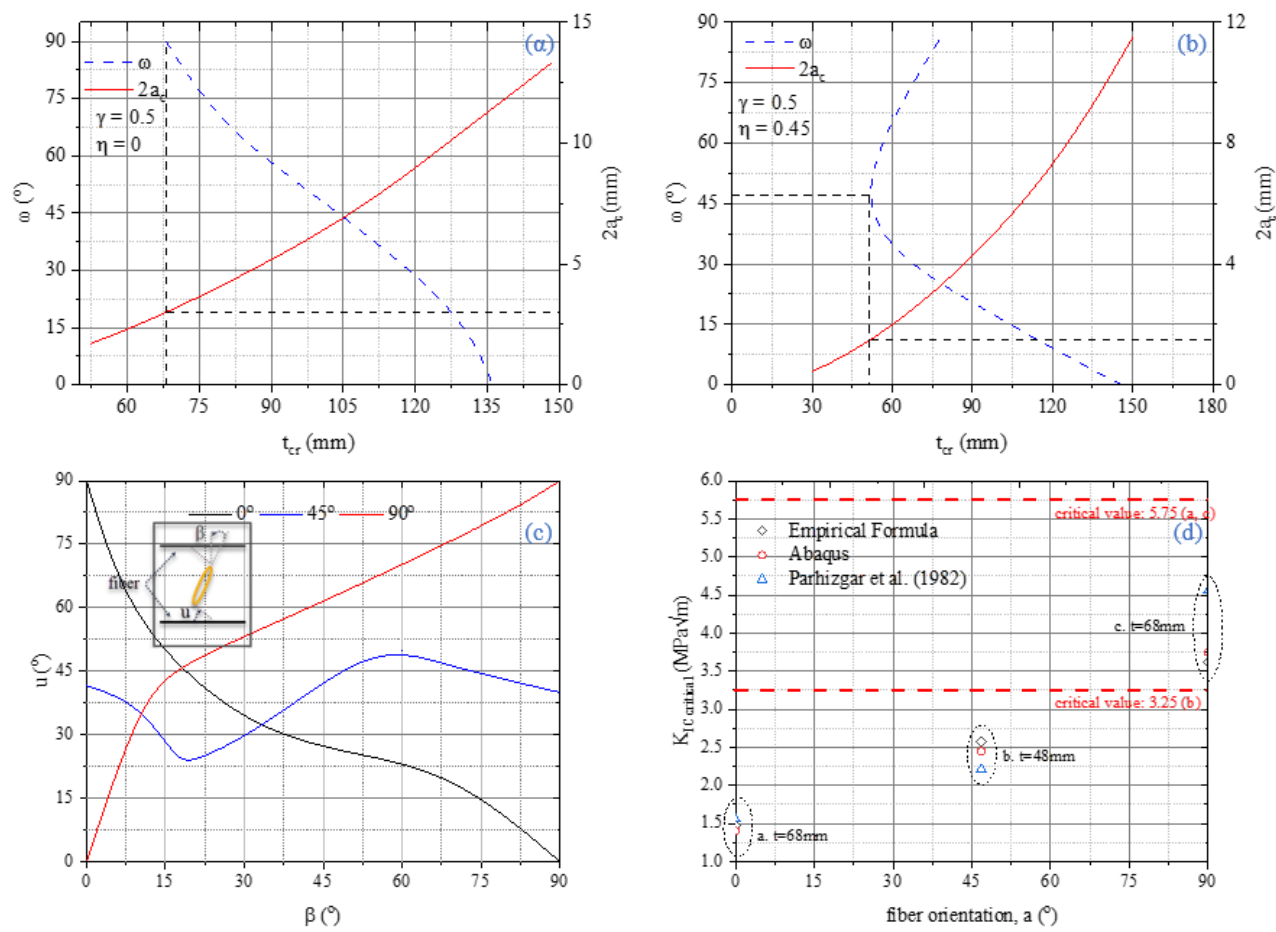

Fig. 3. Analytical results for a) internal pressure, b) combined loading, c) crack growth for different $\omega$ angles (d) and comparison of SIF among analytical, X-FEM and Exp. results.
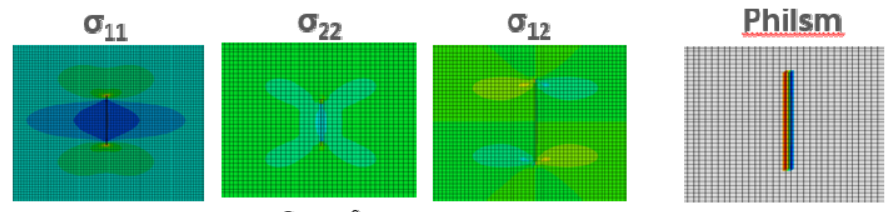

Psilsm
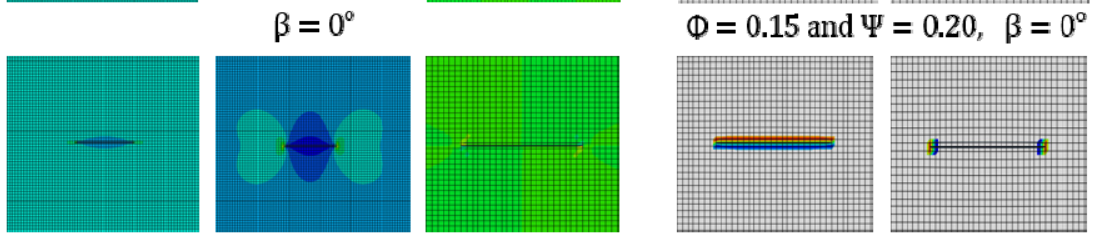

$\beta=90^{\circ}$
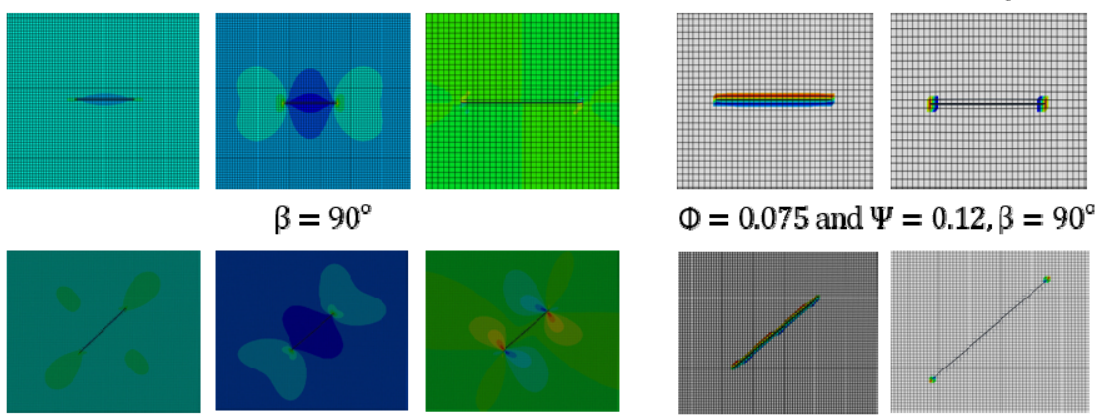

$$
\beta=46.50^{\circ}
$$

(a)

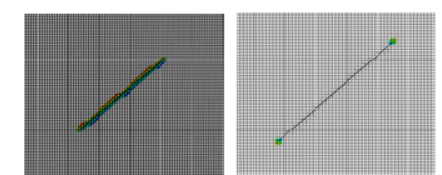

$\Phi=0.034$ and $\Psi=0.018, \beta=46.50^{\circ}(\mathrm{b})$

Fig. 4. Finite element analysis in a sub domain a) Stress state and (b) X-FEM results for $0^{\circ}$, $90^{\circ}$ and $46.50^{\circ}$ respectively.

According to the analytical and FEM results (figure 2), the length of the cracks is not 
dangerous for both cases. It is observed that, crack surfaces opened, but the crack size did not increase.

That means, the stress intensity factors are less than the critical theoretical one. Specifically, the analytical solution of SIF for the first case (thickness $t_{c r}=6.8 \mathrm{~cm}, \beta=0^{\circ}, 90^{\circ}$, and $2 \mathrm{a}_{\mathrm{c}}=0.29$ $\mathrm{cm})$ is equal to 1.469 and $3.619 \mathrm{MPam}^{1 / 2}$, while the corresponding critical value is 5.75 $\mathrm{MPam}^{1 / 2}$. In the second case (thickness $\mathrm{t}_{\mathrm{cr}}=4.8 \mathrm{~cm}, \beta=46.8^{\circ}$, and $2 \mathrm{a}_{\mathrm{c}}=0.12 \mathrm{~cm}$ ) the additional value is 2.45 , while the critical value is $3.25 \mathrm{MPam}^{1 / 2}$. Figure $3 \mathrm{~d}$, presents the comparison among analytical, Abaqus and Parhizgar et. al (11) results. This leads to a reduction of the shell wall thickness. By decreasing the shell wall thickness, other failure criterion must be defined, which could be provide separate failure criteria for matrix, fiber and interface, respectively. The advantage of such failure criteria, provides more accurate results (stressstrains), while its clear the failure by fracture of the structure is mainly attributed to dilatation.

\section{Concluding remarks}

Progress in fracture mechanics analysis has given designers and structural engineers new insights into crack propagation and residual strength of structures with cracks. This study determines the critical crack length when shell design is done according to the Tsai-Hill criterion which is normally used for failure control of anisotropic bodies. According to analytical and finite element results the following conclusions can be made:

- Analysis has demonstrated the necessity of introducing a crack in the shell, subject to a mixed-mode strain because of the nature of the material and its loading conditions.

- The SIF results are in a good agreement and in a way, that the thickness of the vessel could be reduced more than the critical one.

- It becomes clear that safe operation of the structure for long life requires inspection during the functioning stage in order to determine residual strength and act for fracture control.

- Sub-modeling has proven to work well with X-FEM and is recommended to handle larger structures. X-FEM simplifies the mesh modeling, thus increasing flexibility while the accuracy is maintained.

\section{References}

1. E.E. Gdoutos, D.A. Zacharopoulos, E.I. Meletis, Eng. Fract. Mech. 34 (1989)

2. D.A. Zacharopoulos, E.E. Gdoutos EE, Proc. 3nd Inter. Symp. Adv. Comp. Emerg. Tech., Patra, Greece, 1990

3. J.H. Underwood, D.P. Kendall, Theor. Appl. Fract. Mech., 2 (1984)

4. B. El Khalil Hachi, S. Rechak, Y. Belkacemi, G. Maurice, Int. J. Pres. Ves. Pip., 82 (2005)

5. H. Adldoost, A. Zabihollah, S.J. Fattahi, http://journaltool.asme.org/PDF/PVT/111042/DRAFT-PVT-11-1042-0.pdf 2011, 1 (2011)

6. C.H. Chue, C.J. Hwang, Comp. Struct., 46(2) (1993)

7. S.G. Lekhnitskii, Gordon and Breach Science Publication, New York. 1968

8. G.C. Sih, P.C. Paris, G.R. Irwin, Int. J. Fract., 1 (1965)

9. G.C. Sih, Eng. Fract. Mech., 5 (1973)

10. G.C. Sih, E.P. Chen: J. Comp. Mat., 9 (1975)

11. S. Parhizgar, L.W. Zachary, C.T. Sun, Fract. Comp. Mater. (Ed. Sih G.C. and Tamuzs V.P.), Martinus Nijhoff Publication; 1982 